Le Lait, 1986, 66 (3), 189-206

\title{
Utilisation du lysozyme en tant qu'agent régulateur de l'affinage en fromagerie MMV
}

\author{
H. GOUDEDRANCHE, P. DUCRUET, J.-C. VACHOT, R. PANNETIER \\ et J.-L. MAUBOIS \\ I.N.R.A., Laboratoire de Recherches de Technologie laitière \\ 65 , rue de Saint-Brieuc - 35042 Rennes cedex-France
}

\section{Résumé}

L'évolution du $\mathrm{pH}$, de la flore microbienne et de l'azote non protéique des fromages Camembert et Saint-Paulin issus de technologies classiques et MMV a été comparée au cours de leur affinage. Si aucune différence significative n'était mise en évidence pour ce qui est des fromages Camembert, il n'en était pas de même pour les fromages Saint-Paulin issus des deux technologies. Les fromages fabriqués à partir de lait ultrafiltré contenaient 10 fois plus de germes aérobies mésophiles; leur pH restait plus élevé $(+0,15$ unité $\mathrm{pH})$ et leur teneur en azote soluble était plus faible et ce, tout au long de la période d'affinage. Leurs qualités organoleptiques étaient considérées comme légèrement inférieures à celles des fromages traditionnels. L'origine de ces observations est à rechercher dans l'élévation du pouvoir tampon des pâtes découlant de la surminéralisation calcique liée à l'ultrafiltration au $\mathrm{pH}$ du lait.

L'addition de lysozyme aux préfromages Saint-Paulin et Camembert à la dose de $0,5 \mathrm{~g}$ à $1,0 \mathrm{~g}$ par $\mathrm{kg}$ semble être un moyen intéressant pour accélérer et diriger l'affinage des fromages issus de la technologie MMV. Une telle addition réalisée après acidification lactique des préfromages Saint-Paulin conduisait à des évolutions du $\mathrm{pH}$, de la flore aérobie mésophile, de la protéolyse et par voie de conséquence des qualités organoleptiques des fromages de lait ultrafiltré, similaires à celles observées dans les fromages traditionnels. La flaveur et la texture des fromages Camembert issus de technologie MMV étaient également améliorées.

L'ensemble des résultats obtenus conduit à penser à une décomplexation progressive du lysozyme actif à partir des micelles de caséine au cours de l'affinage des pâtes fromagères, hypothèse émise par THAPON et BRULÉ (1986). Les possibilités d'emploi du lysozyme comme enzyme d'affinage en fromagerie classique et MMV sont discutées.

Mots clés : Fromagerie - Saint-Paulin - Camembert - Ultrafiltration - Lysozyme Affinage.

\section{Summary}

Use of lysozyme for managing ripening of cheeses made through MMV process

During ripening time, evolution of $\mathrm{pH}$, total mesophilic flora and NPN content was checked in Saint-Paulin (semi-hard) and Camembert (soft) cheeses made through 
MMV and classical technologies. No significant difference was observed in Camembert cheeses made by both processes but Saint-Paulin cheeses made from ultrafiltrated milk had a higher mesophilic flora, a higher $\mathrm{pH}$ and a lower NPN content for all the period of ripening. Consequently, their organoleptic qualities were judged as sligthly inferior to these of cheeses made through conventional processes. These observations are probably the consequences of the increase of buffering capacity of UF precheeses, increase due to the concentration of calcium salts bound to casein micelle by UF.

Addition of lysozyme to Saint-Paulin and Camembert precheeses (at 5 or $1,0 \mathrm{~g} / \mathrm{kg}$ ) seems to be an interesting mean for accelerating and for managing ripening of cheeses made through MMV process. Such an addition realized after lactic acidification of Saint-Paulin precheeses led to evolution of $\mathrm{pH}$, of mesophilic bacterial count and of proteolysis during ripening and consequently, of organoleptic qualities in UF cheeses similar to these of traditional cheeses. Taste, flavour and texture of UF Camembert cheeses were also improved.

From the results obtained in this study, it seems very likely that lysozyme is progressively decomplexed from casein micelles as active enzyme during ripening as recently suggested by THAPON and BRULÉ (1986).

Possible uses of lysozyme as ripening agent in classical and MMV cheesemaking are discussed.

Key words : Cheesemaking - Saint-Paulin - Camembert - Ultrafiltration - Lysozyme Ripening.

\section{Introduction}

Il est possible d'obtenir un large éventail de textures dans les pâtes fromagères lors de la mise en œuvre de la technique d'ultrafiltration, par action sur le lait avant ou pendant la mise en contact avec les membranes (maturation lactique, diafiltration pour ajuster la teneur en lactose, addition de $\mathrm{NaCl}$ ), par action sur le préfromage liquide final ou intermédiaire (traitement thermique, homogénéisation, addition de chlorure de sodium, de citrate, ou bien d'enzymes d'affinage telles que la « rulactine ») ou enfin par action sur les paramètres classiques de transformation en fromage (conduite de l'acidification, nature du levain utilisé, dose de présure, $\mathrm{pH}$ d'emprésurage, conduite du saumurage et de l'affinage).

Par contre et bien que peu d'analyses sensorielles rigoureuses aient été faites sur ce sujet, il a souvent été reproché aux fromages issus de technologie M.M.V. (Maubois, Mocquot et VAssal, 1969) de manquer, à l'état jeune, de flaveur par rapport aux produits similaires issus de technologie classique. Cette observation a été faite presque exclusivement pour des types de fromages traditionnels où il y avait utilisation de levains lactiques mésophiles : Camembert, pâtes molles de chèvre ou Saint-Paulin. Il semblerait au contraire que ce manque de flaveur à l'état jeune ne soit pas noté quand il y a emploi de levains lactiques thermophiles (Ducruet et al., 1981 ; brevet Guilloteau, 1981 ; Vassal, 1984).

La saveur et l'arôme deviennent dans les deux à trois premières semaines d'affinage, pour les pâtes fromagères issues de la technologie d'ultrafiltration, similaires à ceux des produits traditionnels, mais il n'en reste pas moins que ce manque de flaveur, à l'état jeune, restreint la fenêtre de commercialisation et qu'aussi bien son origine que des palliatifs doivent être recherchés. 
Etant donné le peu de connaissances disponibles et la complexité des mécanismes de formation de la flaveur des fromages, il est particulièrement hasardeux d'émettre des hypothèses quant à l'origine de ce manque de saveur et d'arôme à l'état jeune des fromages issus de lait ultrafiltré. Les questions qui peuvent, néanmoins, être formulées sont les suivantes : le passage au travers de la membrane des petites molécules azotées (peptides, acides aminés libres, urée) et des acides gras libres à courte chaîne amène-t-il à une insuffisance en facteurs de croissance ou en précurseurs de flaveurs, insuffisance qui devrait être palliée par le métabolisme bactérien ultérieur? Le pouvoir tampon plus élevé des pâtes fromagères M.M.V. dû à leur minéralisation supérieure, bien qu'elle puisse être corrigée (BRULÉ et al., 1974), entraîne-t-il une « survie » des cellules des bactéries lactiques donc une lyse moindre qui retarde d'autant la libération des enzymes intracellulaires dans les pâtes fromagères?

Si cette dernière hypothèse était exacte, elle pouvait être vérifiée par addition aux préfromages soit de lysats bactériens, soit d'agents à même de provoquer cette lyse des cellules de bactéries lactiques. Un tel agent pourrait être le lysozyme (E.C. 3.2.1.17.) dont l'emploi est autorisé en France depuis 1981 à la dose maximale de $35 \mathrm{ppm}$ pour la fabrication de fromages à pâte pressée cuite.

L'objet du présent travail était d'une part de comparer l'évolution du $\mathrm{pH}$, de la flore microbienne et de l'azote non protéique des fromages Camembert et Saint-Paulin issus de technologies classiques et M.M.V. et d'autre part d'étudier l'évolution de ces mêmes caractéristiques biochimiques et du développement de la flaveur dans des pâtes fromagères provenant de préfromages auxquels avaient été additionnés des levains « lysozymés » ou du lysozyme en quantité variable.

\section{Matériels et méthodes}

\section{A. Fabrication des fromages}

\section{Fromages Saint-Paulin}

a) Saint-Paulin conventionnel

Les fromages fabriqués selon Ia technologie propre à l'atelier Préval (35360 Montauban-de-Bretagne) étaient prélevés en sortie de saumurage et affinés à $13-14^{\circ} \mathrm{C}$ avec enrobage le $5^{\mathrm{e}}$ jour.

b) Saint-Paulin M.M.V.

Le lait utilisé était un lait de grand mélange, à teneur en matière grasse ajustée $(28 \mathrm{~g} / \mathrm{kg})$, pasteurisé à $80^{\circ} \mathrm{C}, 15 \mathrm{~s}$. Il était ultrafiltré sur un appareillage S.F.E.C. comportant 2 étages ayant respectivement des surfaces membranaires de 6,8 et de $1,6 \mathrm{~m}^{2}$, à une température de $50^{\circ} \mathrm{C}$. Lorsque le facteur de concentration en volume (FCV) 3 était atteint, la dilution de la phase aqueuse était réalisée par addition d'un volume d'eau à $50^{\circ} \mathrm{C}$ égal à 95 p. 100 du volume de rétentat FCV 3. L'ultrafiltration était poursuivie jusqu'à obtention d'un rétentat contenant $45 \mathrm{~g}$ pour $100 \mathrm{~g}$ de matière sèche. Les rétentats étaient immédiatement refroidis à $30^{\circ} \mathrm{C}$, additionnés de levains lactiques mésophiles classiques industriels 
à la dose de $2 \mathrm{~g}$ pour $100 \mathrm{~g}$ et de chlorure de sodium à la dose de $0,5 \mathrm{~g}$ pour $100 \mathrm{~g}$, maintenus à cette température pendant $18 \mathrm{~h}$ et divisés en parties aliquotes.

Des parties aliquotes étaient additionnées de levains lysozymés soit mésophiles ( $4 \mathrm{~g}$ pour $100 \mathrm{~g}$ de préfromage), soit thermophiles ( $3 \mathrm{~g}$ pour $100 \mathrm{~g}$ de préfromage) obtenus comme suit :

- levain lysozymé mésophile : $1 \mathrm{~g}$ de lysozyme en poudre (AFILACT), préalablement dissous dans une petite quantité d'eau, était mélangé à 11 de levain lactique mésophile industriel contenant $1,1.10^{8}$ germes/g. La solution était maintenue à $37^{\circ} \mathrm{C}$ pendant $30 \mathrm{~min}$. Ce traitement n'influençait guère la population bactérienne dénombrable qui était alors de $0,9.10^{8}$ germes/g, puis refroidi à $15^{\circ} \mathrm{C}$ pour conservation (au maximum $24 \mathrm{~h}$ ) avant utilisation. Les fromages correspondant aux préfromages additionnés de ce levain lysozymé étaient dénommés MMVm ;

- levain lysozymé thermophile: les cellules bactériennes contenues dans un levain thermophile industriel (fromagerie d'Emmental) étaient concentrées 3 fois en volume par centrifugation $(1000 \mathrm{~g})$. Au culot de centrifugation $\left(0,7.10^{9} \mathrm{germes} / \mathrm{g}\right)$, il était mélangé $3 \mathrm{~g}$ pour $1000 \mathrm{~g}$ de lysozyme préalablement dissous. Le mélange était maintenu à $42^{\circ} \mathrm{C}$ pendant 60 min puis refroidi à 5-6 ${ }^{\circ} \mathrm{C}$ pour conservation (au maximum $24 \mathrm{~h}$ ) avant utilisation. Là encore, la population bactérienne ne décroissait pas, au contraire puisque $2,3.10^{9}$ germes/g étaient dénombrés. Les fromages correspondant aux préfromages additionnés de ce levain lysozymé étaient dénommé MMV th.

D'autres parties aliquotes recevaient directement du lysozyme $(0,5 \mathrm{~g}$ ou $1 \mathrm{~g}$ pour $1000 \mathrm{~g}$ de préfromage). Les fromages correspondants étaient dénommés MMV L05 et MMV L1.

Les autres parties aliquotes de préfromage servaient de témoins. Les fromages correspondants étaient dénommés MMV.

L'ensemble des préfromages ainsi obtenus était emprésuré $(20 \mathrm{ml}$ de présure liquide commerciale pour $100 \mathrm{~kg}$ de préfromage) et versé en moules. Le démoulage s'effectuait au bout de $60 \mathrm{~min}$. Les fromages étaient maintenus à $28-30^{\circ} \mathrm{C}$ pendant 6 à $8 \mathrm{~h}$ puis à $20^{\circ} \mathrm{C}$ pendant $15 \mathrm{~h}$, salés en saumure saturée à $12^{\circ} \mathrm{C}$ (120 min), séchếs (1 journée) et mis en cave d'affinage $\left(12^{\circ} \mathrm{C}\right)$. Ils étaient enrobés de cire (cire brune 6409 - Superdex) le $5^{\mathrm{e}}$ ou le $7^{\mathrm{e}}$ jour.

\section{Fromages Camembert}

\section{a) Camembert conventionnel}

Le lait utilisé était du lait industriel, de grand mélange, à teneur en matière grasse ajustée $(28 \mathrm{~g} / \mathrm{kg})$ pasteurisé HTST $\left(72^{\circ} \mathrm{C} ; 15 \mathrm{~s}\right)$, ensemencé en levain lactique mésophile classique $(0,5 \mathrm{~g}$ pour $1000 \mathrm{~g})$, conservé à $10-11^{\circ} \mathrm{C}$ pendant la nuit, réchauffé à $33^{\circ} \mathrm{C}$, de nouveau additionné de $1,3 \mathrm{~g}$ pour $100 \mathrm{~g}$ de levain lactique mésophile et de divers additifs : chorure de calcium $(0,1 \mathrm{ml} / \mathrm{kg}$ d'une solution contenant $520 \mathrm{~g} / 1$ de $\mathrm{CaCl}_{2}$ déshydraté) ; spores de Penicillium camemberti (souche Lacto-labo LV2 : 10 doses commerciales/1 0001 de lait) ; levures (levures d'affinage Lacto/labo DH : 10 doses commerciales/1 0001 de lait), puis réparti en bassines de $85 \mathrm{I}$. Lorsque le $\mathrm{pH}$ atteignait une valeur de 6,3-6,25, $18 \mathrm{ml}$ de solution de présure étaient ajoutés pour 1001 de lait, 35 à 40 min après l'emprésurage, le caillé était découpé et maintenu pour durcissement pendant cette même durée. Le lactosérum surnageant était alors décanté et le caillé 
réparti en moules. La température de la salle d'égouttage était maintenue à $27-28^{\circ} \mathrm{C}$ pendant $4 \mathrm{~h}$ puis progressivement ramenée à $23^{\circ} \mathrm{C}$. Le jour suivant, les fromages étaient salés en saumure saturée à $12^{\circ} \mathrm{C}(30 \mathrm{~min})$, séchés $24 \mathrm{~h}$ et placés en haloirs à $12^{\circ} \mathrm{C}$. Le $10^{\mathrm{e}}$ jour, ils étaient emballés. Le $15^{\mathrm{e}}$ jour, ils étaient placés en enceinte réfrigérée à $6-10^{\circ} \mathrm{C}$.

\section{b) Camembert MMV}

Le même lait que celui utilisé pour la fabrication des Camemberts conventionnels était réchauffé à $30^{\circ} \mathrm{C}$, additionné du même levain lactique mésophile $(1,2 \mathrm{~g}$ p. $100 \mathrm{~g})$ et soumis à l'ultrafiltration soit sur l'équipement SFEC décrit ci-dessus, soit sur un équipement ROMICON comportant $5 \mathrm{~m}^{2}$ de membranes PM 50. Au FCV 3, il était procédé à une diafiltration (addition d'un volume d'eau égal à 11 p. 100 du volume du rétentat FCV 3). La concentration était poursuivie jusqu'à obtention d'un rétentat contenant $34 \mathrm{~g}$ p. $100 \mathrm{~g}$ de matière sèche. Le $\mathrm{pH}$ du rétentat final était de 6,4 . Après addition de $0,5 \mathrm{~g} \mathrm{p} .100 \mathrm{~g}$ de chlorure de sodium, le rétentat était maintenu à $30^{\circ} \mathrm{C}$ jusqu'à obtention d'un $\mathrm{pH}$ de 5,2 . Il était alors additionné de $P$. camemberti ( 2 doses/100 1) et de levures ( 2 doses/ $100 \mathrm{l})$. Des parties aliquotes de ce préfromage étaient additionnées, soit de $0,5 \mathrm{~g}$ p. $1000 \mathrm{~g}$ (fromages correspondants dénommés MMV L05), soit de $1 \mathrm{~g} \mathrm{p} .1000 \mathrm{~g}$ de lysozyme (fromages correspondants dénommés MMV L1). D'autres servaient de références (fromages correspondants dénommés MMV). L'ensemble était emprésuré $(20 \mathrm{ml}$ de solution de présure $100 \mathrm{~kg}$ de préfromage), moulé puis démoulé après $1 \mathrm{~h}$. Les fromages étaient maintenus $2 \mathrm{~h}$ à $27-28^{\circ} \mathrm{C}$ puis $16 \mathrm{~h}$ à $25^{\circ} \mathrm{C}$ avant d'être salés, séchés, mis en haloir puis en enceinte réfrigérée comme indiqué pour les fromages conventionnels.

\section{B. Déterminations analytiques}

La teneur en substance sèche (ES) des rétentats et du fromage était dêterminée par dessication à $102^{\circ} \mathrm{C}$ durant $7 \mathrm{~h}$. La teneur en matière azotée totale (MAT) était déterminée à l'aide de l'appareillage «Auto-analyser Technicon II » après dissolution des échantillons dans une solution de citrate à $2 \mathrm{~g}$ pour $100 \mathrm{~g}$, La teneur en azote non protéique (NPN) était déterminée par la méthode suivante. Les échantillons de fromage étaient solubilisés dans une solution de soude $0,2 \mathrm{~N}$ à $45^{\circ} \mathrm{C}$. Après dissolution du fromage, $40 \mathrm{ml}$ d'une solution de TCA à $15 \mathrm{~g}$ p. $100 \mathrm{~g}$ étaient ajoutés à $10 \mathrm{ml}$ de solution de fromage de façon à obtenir une concentration finale en TCA de $12 \mathrm{~g} \mathrm{p} .100 \mathrm{~g}$. Après filtration, l'azote du filtrat était déterminé par la méthode utilisée pour le dosage de l'azote total. Le $\mathrm{pH}$ était mesuré à 0,02 unité de $\mathrm{pH}$ à l'aide d'un appareillage « Tacussel Minisis 5000 » par lecture directe des échantillons de lait, rétentat, préfromage et fromage après broyage et malaxage de l'échantillon (croûte comprise). La numération de la flore totale était effectuée soit directement (levains), soit après dissolution du préfromage ou du fromage dans une solution stérile de citrate de sodium à $2 \mathrm{~g}$ p. $100 \mathrm{~g}$ par la méthode des dilutions décimales en boîtes de Petri renfermant un milieu gélosé nutritif (Plate Count Agar Difco $n^{\circ}$ 042001). Les boîtes étaient incubées : $30^{\circ} \mathrm{C}$ pendant 3 jours pour le dénombrement de la flore mésophile, à $37^{\circ} \mathrm{C}$ pendant 2 jours pour le dénombrement de la flore thermophile. L'analyse sensorielle des fromages était effectuée par un jury composé de membres qualifiés du laboratoire et de fromagers spécialistes de ces types de fromages. 


\section{Résultats}

\section{A. Composition des fromages}

La composition des fromages obtenus est indiquée dans le tableau 1.

\section{TABLEAU I}

Composition des fromages

Chaque $n^{\circ}$ de fromages pour chaque type de fromage (St-Paulin ou Camembert) correspond à une même journée de fabrication

Cheese composition

Each cheese $n^{\circ}$ for each type of cheese (St-Paulin or Camembert) corresponds to the same day of production

\begin{tabular}{|c|c|c|c|c|c|}
\hline & & $2^{\mathrm{e}}$ jour & & $15^{\mathrm{e}}$ jour & \\
\hline & & $\begin{array}{c}\text { ES } \\
\text { g p. } 100 \mathrm{~g}\end{array}$ & $\begin{array}{c}\text { ES } \\
\text { g p. } 100 \mathrm{~g}\end{array}$ & $\begin{array}{c}\text { MAT } \\
\text { g p. } 100 \mathrm{~g}\end{array}$ & $\underset{\mathrm{ES}}{\mathrm{MG} /}$ \\
\hline \multicolumn{6}{|l|}{ St-Paulin } \\
\hline $\begin{array}{l}\text { Conventionnel } \\
\text { Conventionnel } \\
\text { MMV } \\
\text { MMV } \\
\text { MMV } \\
\text { MMV m } \\
\text { MMV th } \\
\text { MMV L1 } \\
\text { MMV L1 } \\
\text { MMV L1 } \\
\text { MMV L05 }\end{array}$ & $\begin{array}{ll}\mathrm{n}^{\circ} & 1 \\
\mathrm{n}^{\circ} & 2 \\
\mathrm{n}^{\circ} & 1 \\
\mathrm{n}^{\circ} & 2 \\
\mathrm{n}^{\circ} & 3 \\
\mathrm{n}^{\circ} & 1 \\
\mathrm{n}^{\circ} & 2 \\
\mathrm{n}^{\circ} & 2 \\
\mathrm{n}^{\circ} & 3 \\
\mathrm{n}^{\circ} & 4 \\
\mathrm{n}^{\circ} & 4\end{array}$ & $\begin{array}{l}51,0 \\
50,9 \\
48,2 \\
49,1 \\
49,0 \\
47,1 \\
48,7 \\
48,2 \\
47,7 \\
47,4 \\
48,0\end{array}$ & $\begin{array}{l}51,7 \\
51,2 \\
49,9 \\
49,3 \\
51,4 \\
49,9 \\
48,9 \\
48,4 \\
49,6 \\
55,8 \\
55,3\end{array}$ & $\begin{array}{l}21,8 \\
22,0 \\
22,7 \\
21,3 \\
23,8 \\
21,8 \\
21,7 \\
21,0 \\
22,5 \\
- \\
-\end{array}$ & $\begin{array}{l}0,46 \\
0,47 \\
0,43 \\
0,44 \\
0,46 \\
0,43 \\
0,44 \\
0,44 \\
0,46 \\
0,46 \\
0,47\end{array}$ \\
\hline \multicolumn{6}{|l|}{ Camemberts } \\
\hline $\begin{array}{l}\text { Conventionnel } \\
\text { MMV } \\
\text { MMV } \\
\text { MMV L1 } \\
\text { MMV L1 } \\
\text { MMV L05 }\end{array}$ & $\begin{array}{ll}\mathrm{n}^{\circ} & 2 \\
\mathrm{n}^{\circ} & 1 \\
\mathrm{n}^{\circ} & 2 \\
\mathrm{n}^{\circ} & 1 \\
\mathrm{n}^{\circ} & 2 \\
\mathrm{n}^{\circ} & 2\end{array}$ & $\begin{array}{l}\frac{42,5}{40,4} \\
\frac{-}{40,1} \\
40,4\end{array}$ & $\begin{array}{l}45,5 \\
43,0 \\
42,7 \\
42,9 \\
42,8 \\
43,0\end{array}$ & $\begin{array}{l}- \\
- \\
-\end{array}$ & $\begin{array}{l}0,45 \\
0,42 \\
0,45 \\
0,42 \\
0,45 \\
0,45\end{array}$ \\
\hline
\end{tabular}

B. Comparaison des fromages conventionnels et $M M V$

\section{Evolution $d u p H$}

L'évolution du $\mathrm{pH}$ des fromages conventionnels et MMV, au cours de leur affinage, est représentée sur les figures 1 (Saint-Paulin) et 2 (Camembert). Le pH 


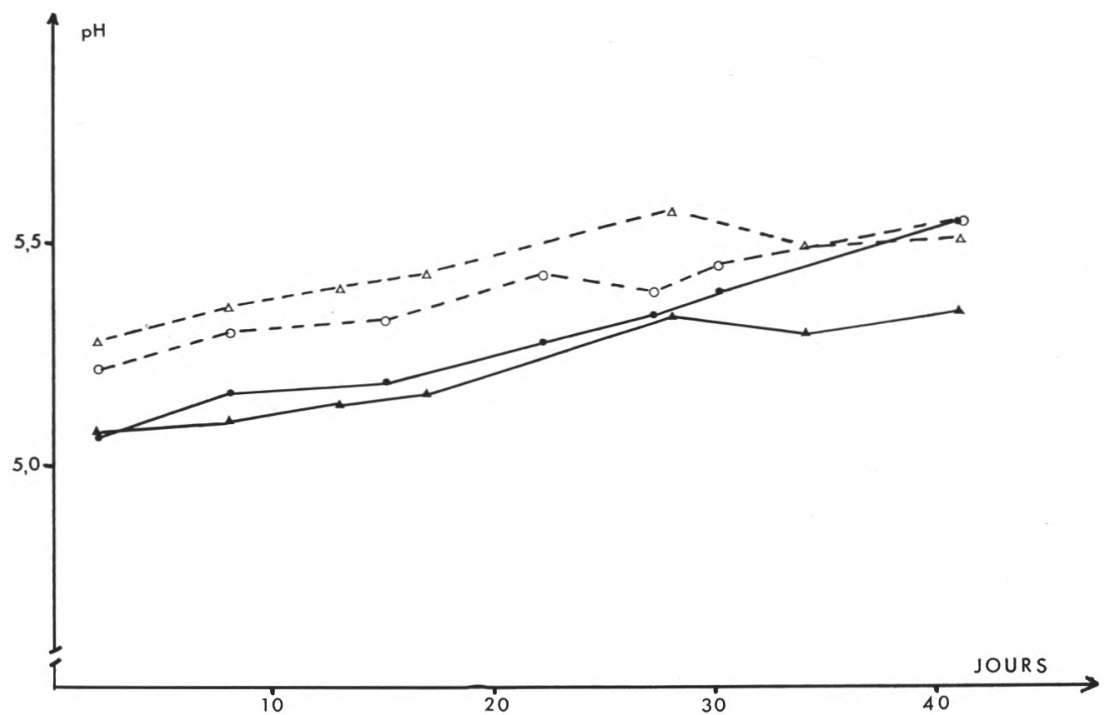

Fig. 1

Evolution $d u p H$ de fromages de type St-Paulin au cours de l'affinage :

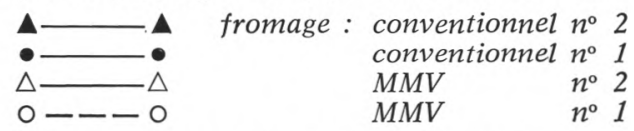

St-Paulin cheese $p H$ evolution during ripening.

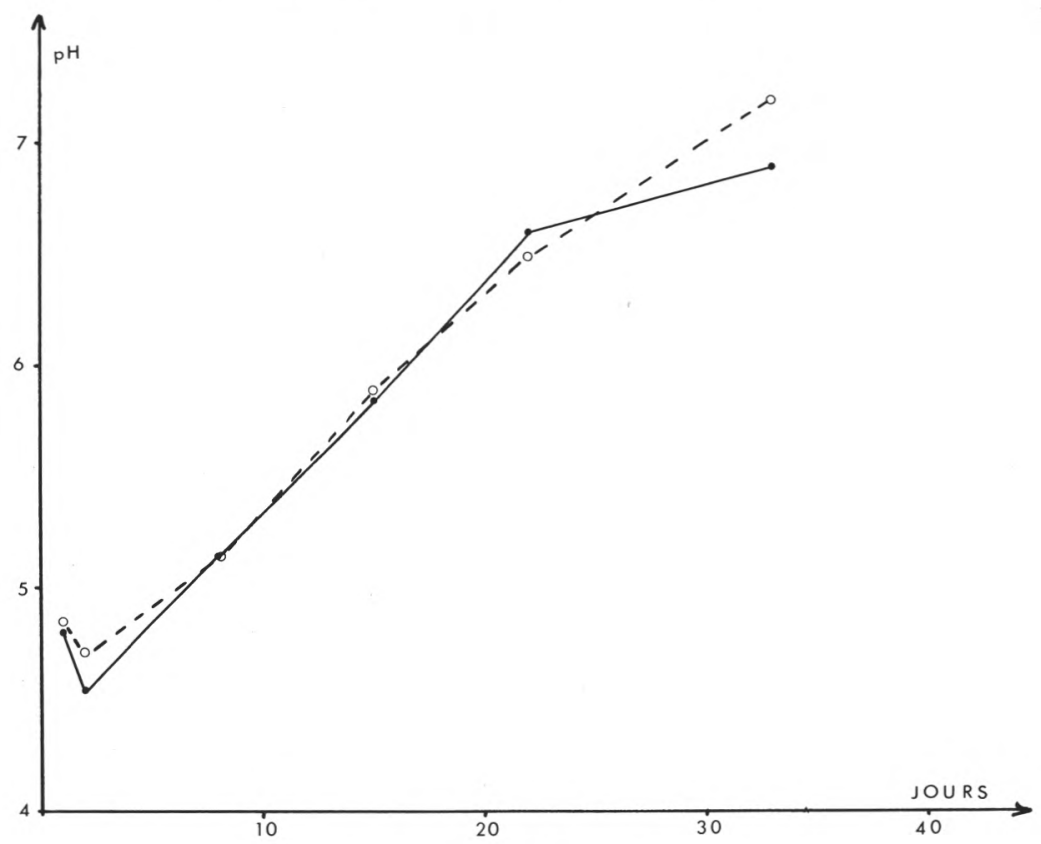

Fig. 2

Evolution $d u$ pH de fromages de type Camembert au cours de l'affinage :

$$
\begin{aligned}
& -\quad \text { fromage : conventionnel } n^{\circ} 2 \\
& \text { O- - } 0 \quad M M V \quad n^{\circ} 2
\end{aligned}
$$

Camembert cheese $p H$ evolution during ripening. 
des Saint-Paulin MMV $(5,20)$ était légèrement plus élevé à $\mathrm{J}+2$ que celui des fromages conventionnels $(5,05)$. Cet écart était conservé jusqu'au $20^{\mathrm{e}}$ jour ; ensuite il avait tendance à s'estomper. Aucune différence significative de $\mathrm{pH}$ n'était constatée pour les fromages Camembert conventionnels et MMV aux différents stades de leur affinage : 4,8 à $\mathrm{J}+1 ; 5,9$ à $\mathrm{J}+15$; environ 7,0 à $\mathrm{J}+33$.

\section{Evolution de la flore aérobie mésophile}

L'évolution de la flore aérobie mésophile des fromages Saint-Paulin conventionnels et MMV est représentée sur la figure 3. Dans les fromages conventionnels, la population bactérienne décroissait de plus d'une puissance de 10 entre le $2^{\mathrm{e}}$ et le $7^{\mathrm{e}}$ jour d'affinage. Elle se stabilisait ensuite à $10^{7}$ germes/g jusqu'au $20^{\mathrm{e}}$ jour pour croître ensuite et atteindre à $\mathrm{J}+45$ une valeur voisine de la population initiale, soit $10^{8}$ germes/g. L'évolution constatée pour les populations des Saint-

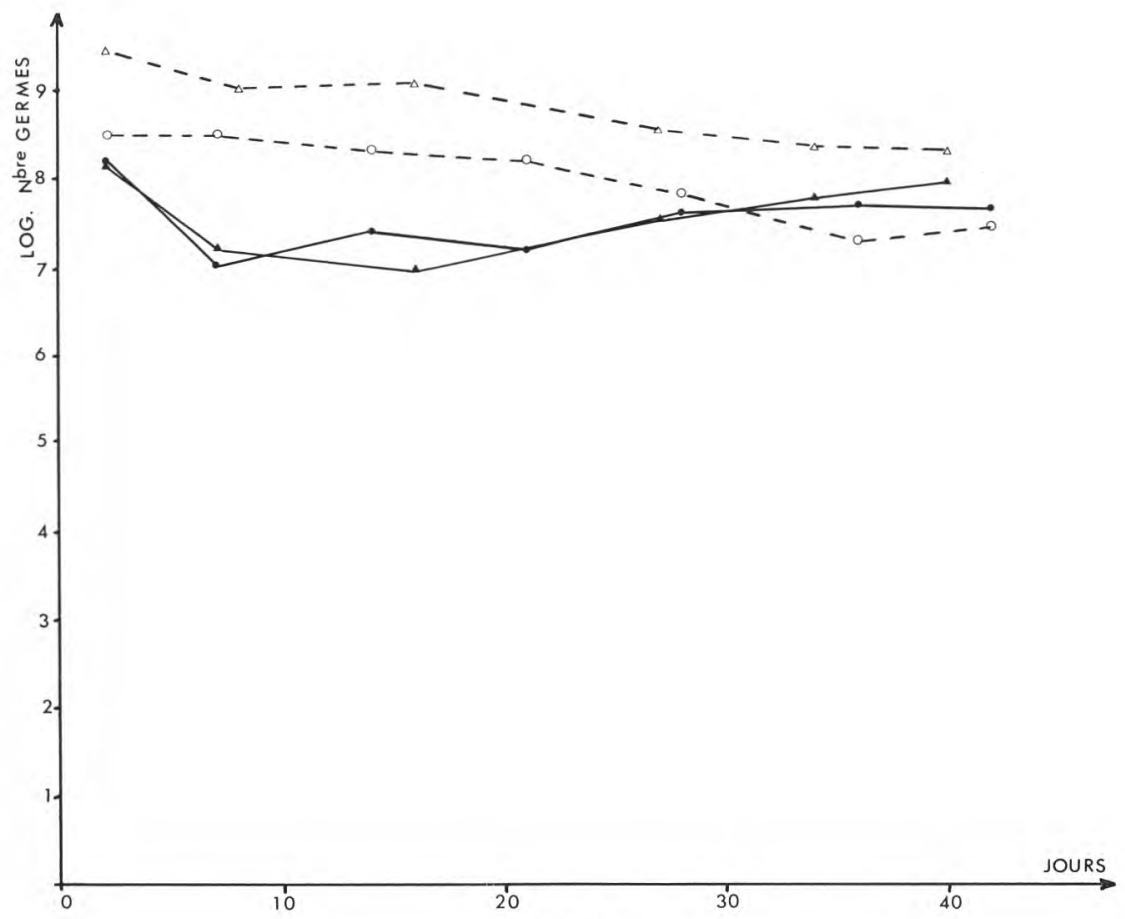

Fig. 3

Evolution du nombre de bactéries contenues dans $1 \mathrm{~g}$ de fromage de type St-Paulin au cours de l'affinage:

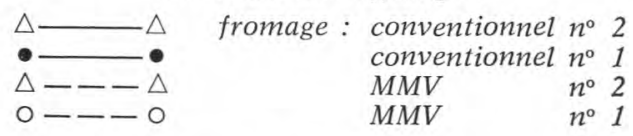

Evolution of aerobic mesophilic total count expressed in CFU/g of cheese during St-Paulin cheese ripening. 
Paulin MMV était différente. La flore à $\mathrm{J}+2$ était supérieure de 0,2 à 1 logarithme. Elle décroissait ensuite régulièrement tout au long de l'affinage. $\mathrm{A} J+35$, les deux types de fromages contenaient le même nombre de germes aérobies mésophiles par g, soit $10^{8}$.

La même comparaison effectuée sur des fromages Camembert conventionnel et MMV (fig. 4) ne mettait en évidence aucune différence significative tout au long de l'affinage. Dans les deux types de fromages, la population était stabilisée à une valeur comprise entre 1 et $5 \times 10^{8}$ germes/g.

3. Evolution de la teneur en azote non protéique

La figure 5 montre l'évolution de la fraction azotée soluble dans le TCA 12 p. 100 des fromages Saint-Paulin au cours de l'affinage. Cette fraction représentait respectivement 2,2 et 4,8 p. 100 de l'azote total des fromages conventionnels et $\mathrm{MMV}$ au $2^{\mathrm{e}}$ jour. Elle augmentait ensuite régulièrement jusqu'en fin

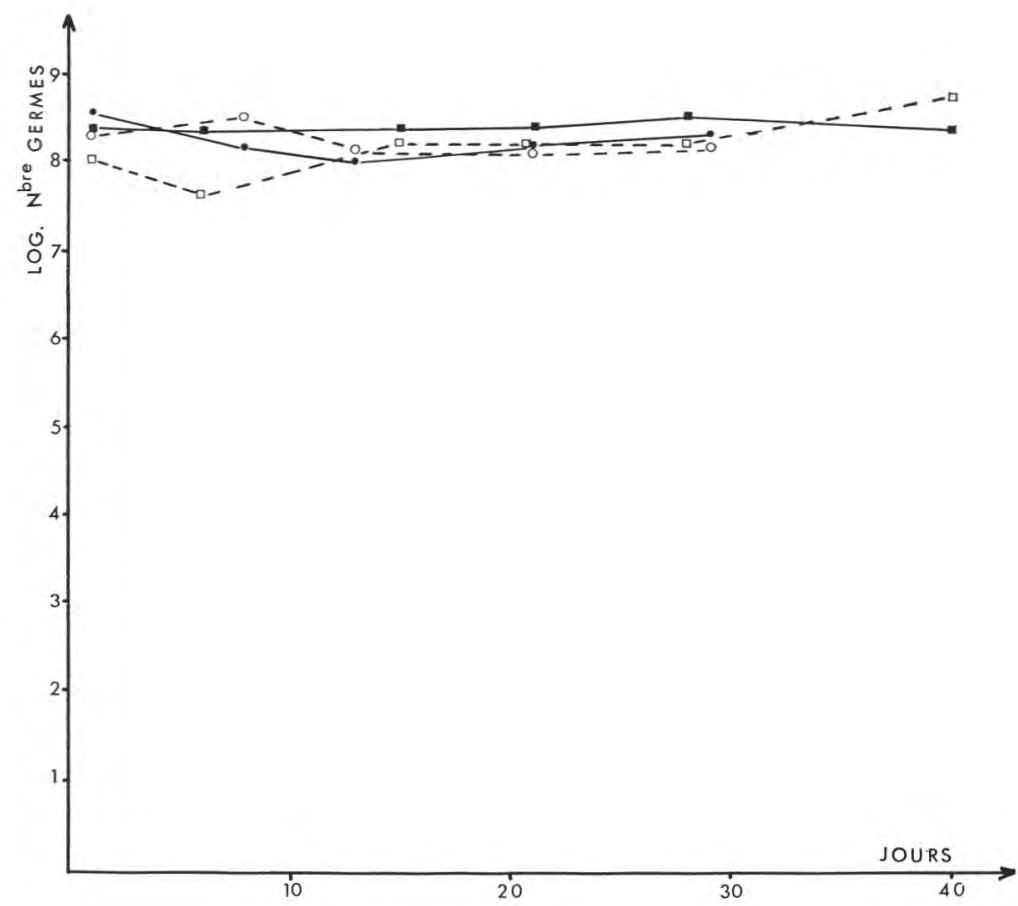

Fig. 4

Evolution of aerobic mesophilic total count expressed in CFU/g of cheese during au cours de l'affinage:

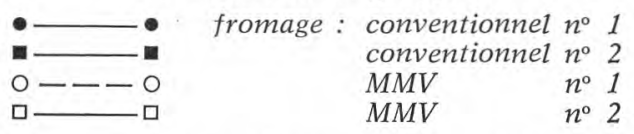

Evolution of aerobic mesophilic total count expressed in CFU/g of cheese during Camembert cheese ripening. 


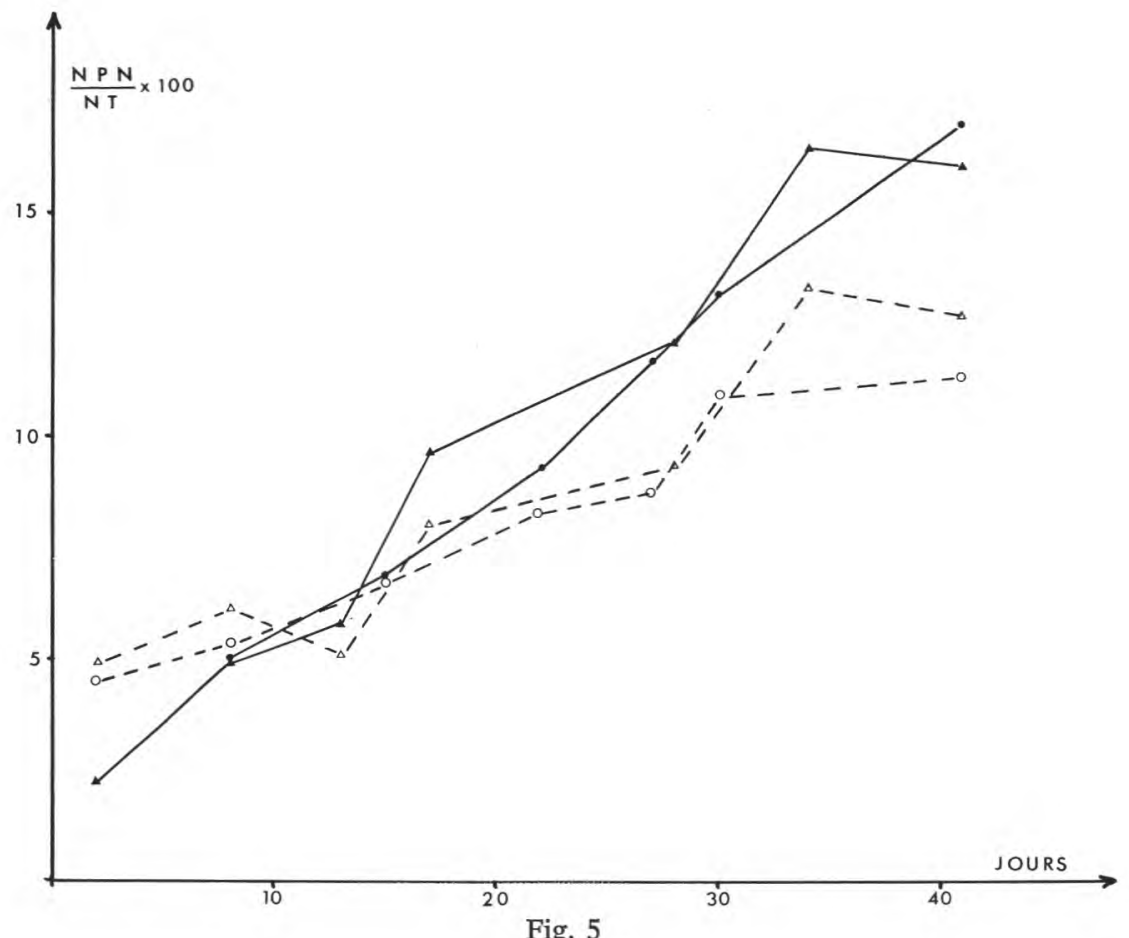

Fig. 5

Evolution de la fraction azotée non protéique (NPN) de fromages de type St-Paulin au cours de l'affinage :

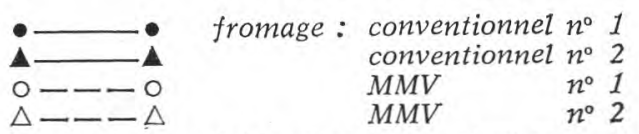

Evolution of the ratio soluble nitrogen/total nitrogen in St-Paulin cheese during ripening.

d'affinage $(J+41)$ mais de façon plus rapide dans. le fromage conventionnel puisqu'elle représentait alors 16 p. 100 de l'azote total contre 12 p. 100 dans le fromage MMV. Entre J +15 et J + 41, cette fraction était d'environ 20 p. 100 supérieure dans les fromages conventionnels par rapport aux fromages MMV.

\section{Addition de levains lysozymés aux préfromages}

L'addition de levains lysozymés mésophile ou thermophile aux préfromages Saint-Paulin ne modifiait ni la valeur $\mathrm{du} \mathrm{pH}$ des fromages à $\mathrm{J}+2$ ni son évolution au cours de l'affinage. Elle ne modifiait pas non plus le nombre de germes présents dans les fromages MMV $m$ et MMV th aux différents stades de l'affinage par rapport aux nombres de germes contenus dans les fromages MMV (fig. 6). Aucune différence n'était constatée non plus en ce qui concerne l'évolution 


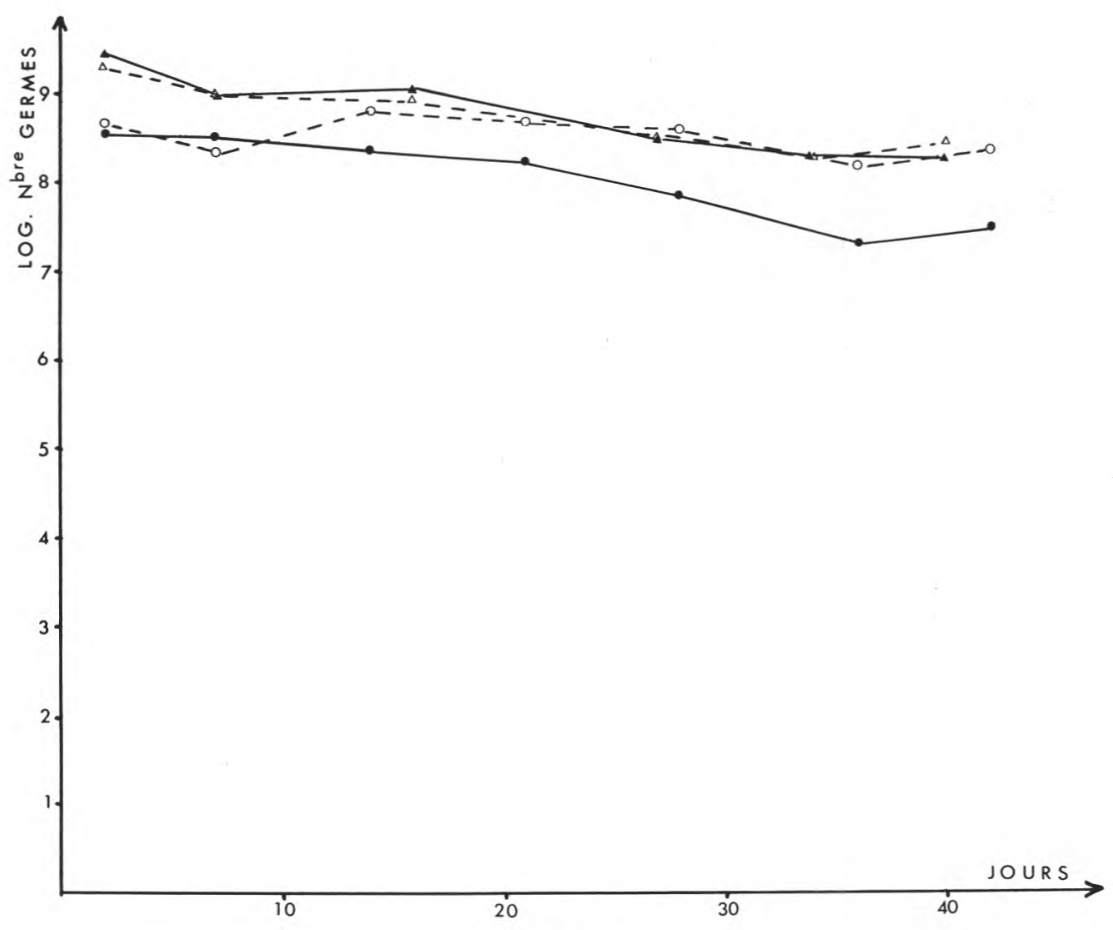

Fig. 6

Evolution du nombre de bactéries contenues dans $1 \mathrm{~g}$ de fromage de type St-Paulin au cours de l'affinage:

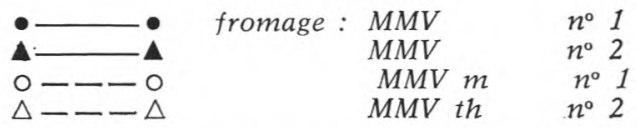

Evolution of aerobic mesophilic total count erpressed in CFU/g of St-Paulin cheeso during ripening.

de la fraction azotée soluble. Il en était de même au cours de la dégustation des trois catégories de fromages.

D. Addition de lysozyme aux préfromages

\section{Evolution du $p H$}

Les fromages MMV et MMV LI Saint-Paulin avaient des $\mathrm{pH}$ identiques du $2^{\mathrm{e}}$ au $41^{\mathrm{e}}$ jour. Les fromages MMV L1 Camembert possédaient un $\mathrm{pH}$ plus élevé de 0,1 à 0,4 unité de $\mathrm{pH}$ entre le $15^{\mathrm{e}}$ et le $30^{\mathrm{e}}$ jour (fig. 7) par rapport à celui des fromages MMV du même type. 


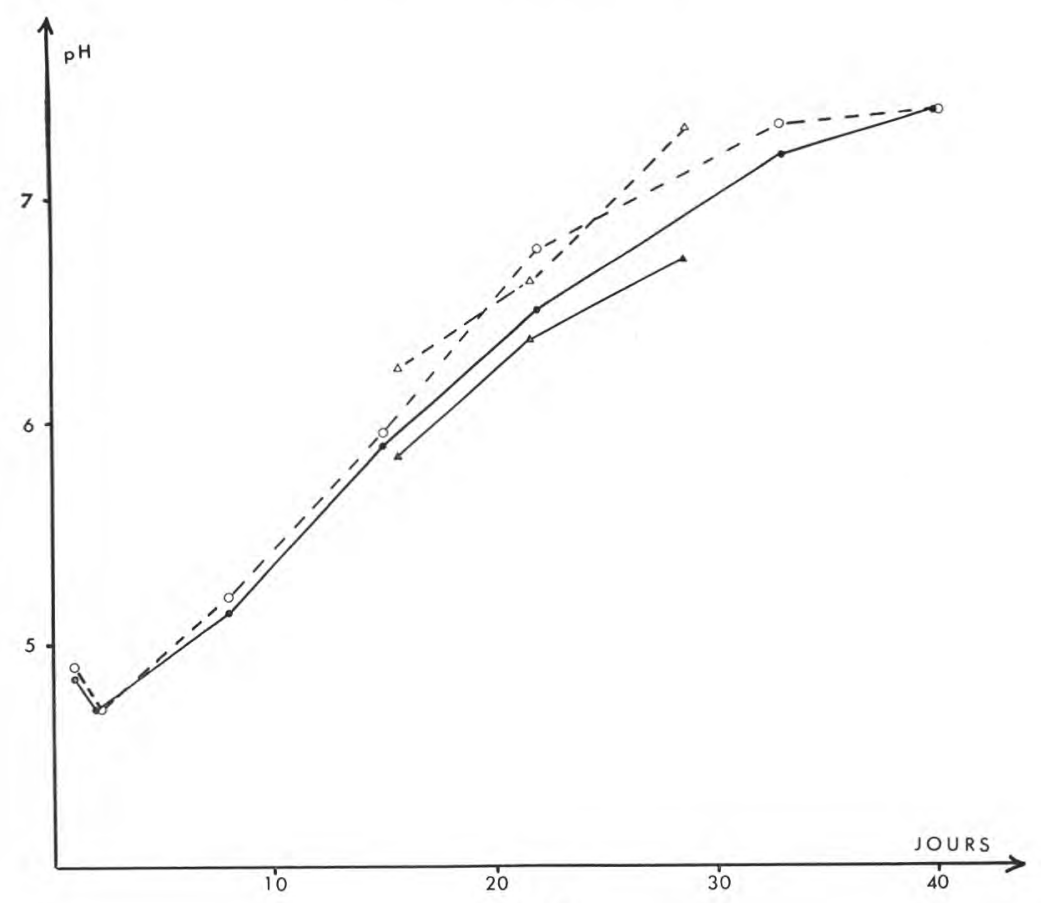

Fig. 7

Evolution du $p H$ de fromages de type Camembert au cours de l'affinage :

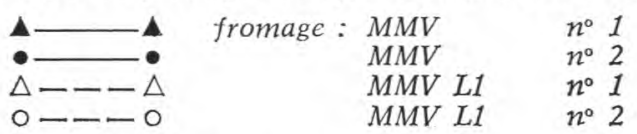

Evolution of Camembert cheese $\mathrm{pH}$ with and without addition of lysozyme during ripening.

\section{Evolution de la flore microbienne}

Le lysozyme ajouté à la dose de $1 \mathrm{~g}$ p. $1000 \mathrm{~g}$ au préfromage modifiait la population bactérienne dans les fromages Saint-Paulin fabriqués par ultrafiltration. Sur la figure 8 est tracée l'évolution du nombre de germes totaux contenus dans 2 fromages MMV et 3 fromages MMV L1. La population bactérienne était toujours inférieure, dans le fromage contenant du lysozyme, à celle du fromage MMV de la même fabrication (MMV L1 ${ }^{\circ} 2$ et MMV $\mathrm{n}^{\circ} 2$; MMV L1 $\mathrm{n}^{\circ} 3$ et MMV $\mathrm{n}^{0} 3$ ). La diminution du nombre de bactéries les premiers jours d'affinage restait cependant inférieure de 0,1 à 1 logarithme suivant les essais à celle observée dans les fromages conventionnels. Une quantité moindre de lysozyme $(0,5 \mathrm{~g}$ p. $1000 \mathrm{~g}$ de préfromage Saint-Paulin) produisait un effet presque similaire (fig. 9). Par contre, l'addition de $1 \mathrm{~g}$ pour $1000 \mathrm{~g}$ de préfromage Camembert ne semblait pas influer sur le nombre de bactéries contenues dans le fromage (fig. 10). 


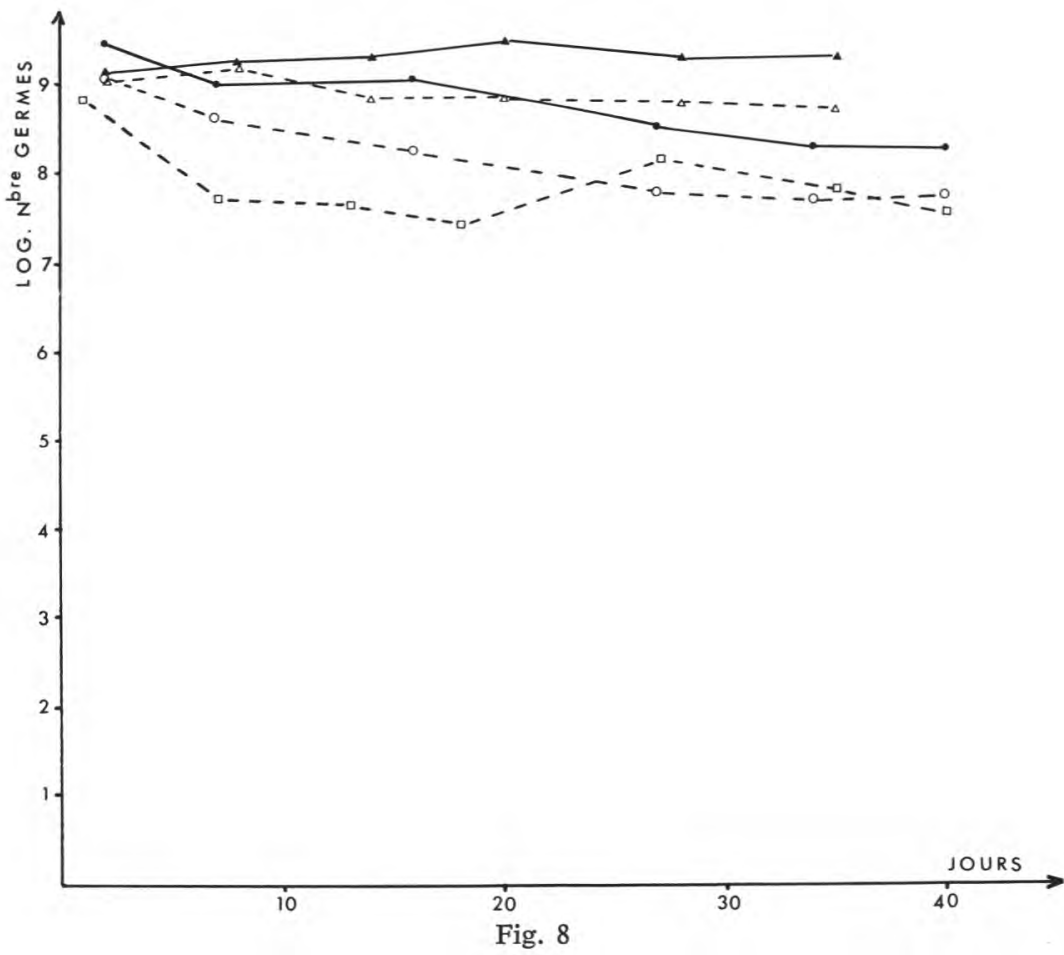

Evolution du nombre de bactéries contenues dans $1 \mathrm{~g}$ de fromage de type St Paulin au cours de l'affinage:

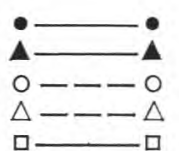

\begin{tabular}{|c|c|}
\hline fromage & $M M V$ \\
\hline & $M M V$ \\
\hline & $M M V L I$ \\
\hline & $M M V L 1$ \\
\hline
\end{tabular}

Evolution of total aerobic mesophilic count per $g$ of St-Paulin cheese with and without addition of lysozyme during ripening.

\section{Evolution de la teneur en azote non protéique}

L'évolution de la fraction azote soluble dans l'azote total des fromages SaintPaulin est représentée dans la figure 11. L'addition de lysozyme aux préfromages entraînait un accroissement de la protéolyse de l'ordre de 13 p. 100 en moyenne à partir du $10^{\mathrm{e}}$ jour d'affinage. Les écarts extrêmes constatés entre les fromages lysozymés ou non variaient entre 7 et 20 p. 100.

\section{Evolution des qualités organoleptiques}

Les textures des fromages Saint-Paulin MMV, MMV L1 et MMV L05 étaient très comparables. Par contre, l'addition de lysozyme aux fromages Camembert issus de technologie MMV améliorait la texture à tous les stades d'affinage. Les fromages MMV L1 et MMV L05 avaient une pâte plus fine et moins collante que celle des fromages MMV, très similaire à celle des fromages conventionnels. 


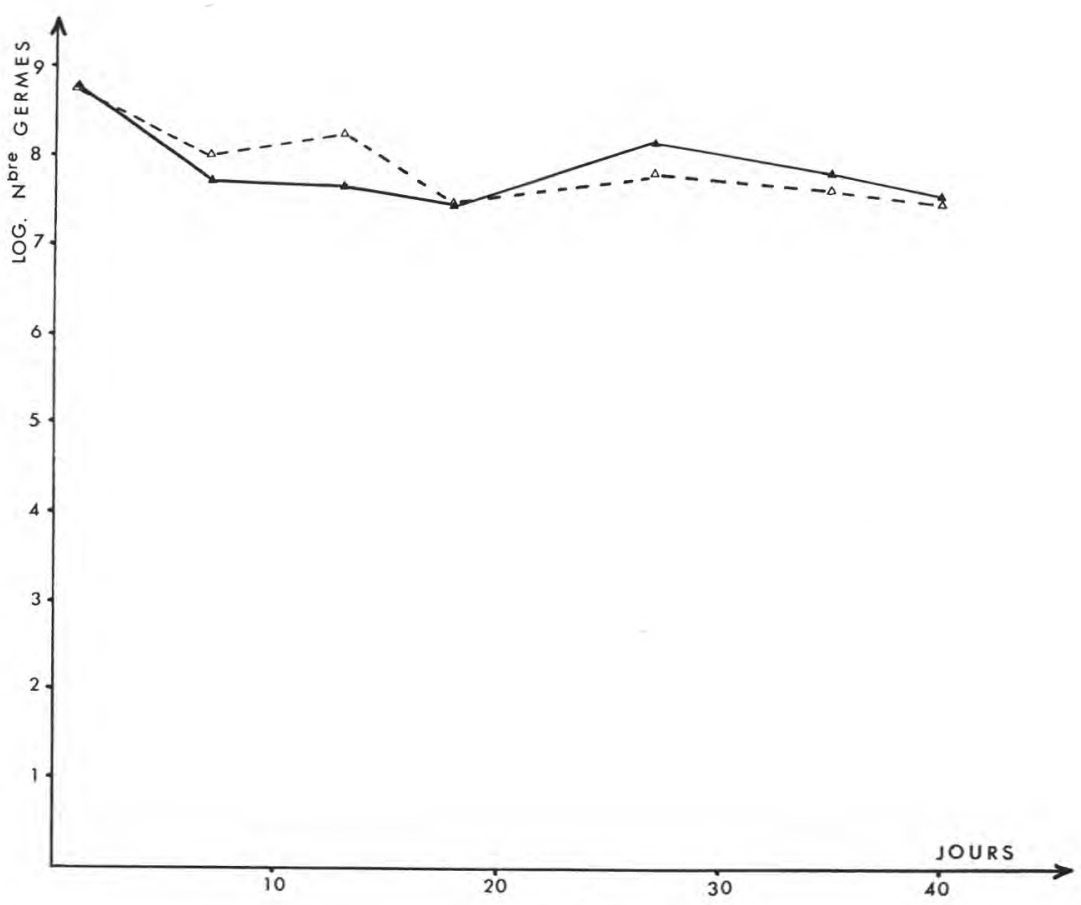

Fig. 9

Evolution du nombre de bactéries contenues dans $1 \mathrm{~g}$ de fromage de type St-Paulin au cours de l'affinage:

$$
\begin{array}{lll}
\Delta-\Delta & \text { fromage : } \\
\triangle M M V L i & n^{\circ} 4 \\
M M V & \text { LO5 } & n^{\circ} 4
\end{array}
$$

Effect of addition of $0.5 \mathrm{~g}$ and $1.0 \mathrm{~g} / \mathrm{kg}$ of precheese on evolution of total aerobic mesophilic count of St-Paulin cheese during ripening.

La comparaison organoleptique des fromages Saint-Paulin MMV et MMV L1 était toujours à l'avantage des seconds. Dès le $10^{\mathrm{e}}$ jour, la flaveur des fromages MMV L1 se rapprochait de celle des fromages conventionnels. L'optimum de goût était atteint vers la fin de la $3^{\mathrm{e}}$ semaine d'affinage, ce goût restant franc jusqu'à 40 jours. Une amélioration de flaveur mais à un degré moindre était également constatée dans les fromages MMV L05. Les fromages Camembert MMV L1 et MMVL05 présentaient une flaveur plus marquée que celle des fromages MMV à partir de $\mathrm{J}+10$ mais ils ne pouvaient être différenciés entre eux. En fin d'affinage, ces deux catégories de fromages malgré leur degré élevé de protéolyse présentaient un caractère moins ammoniacal que les fromages MMV correspondants. Ils pouvaient s'assimiler alors aux Camemberts de lait cru à goût prononcé.

\section{Discussion}

La flore aérobie mésophile des fromages Saint-Paulin obtenus par la technologie MMV est significativement plus élevée en nombre $(1 \mathrm{log})$ que celle des 


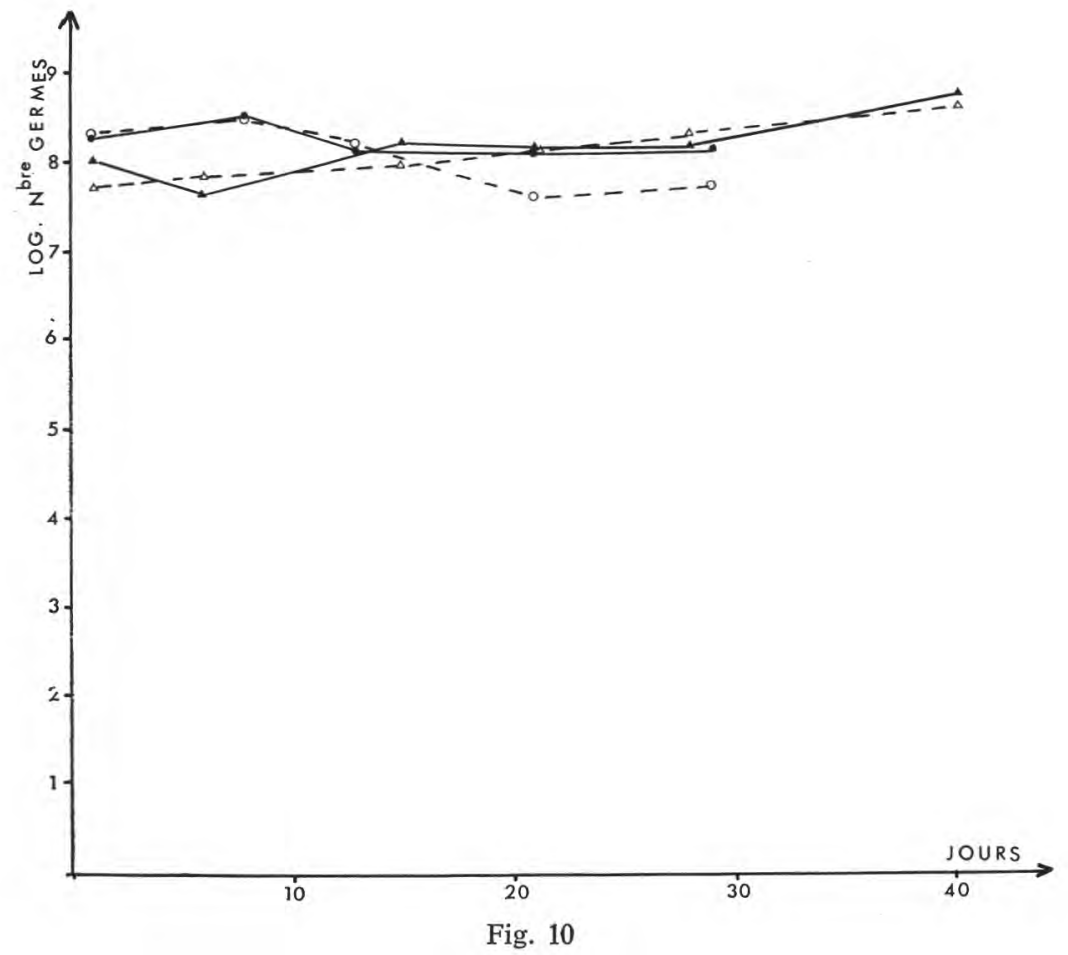

Evolution du nombre de bactéries contenues dans $1 \mathrm{~g}$ de fromage de type Camembert au cours de l'affinage :

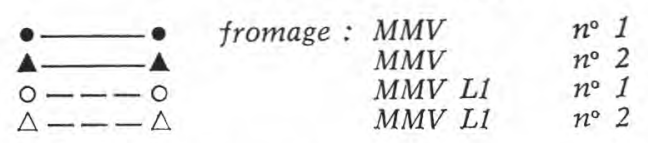

Evolution of total aerobic mesophilic count per $g$ of Camembert cheese added or not with lysozyme during ripening.

fromages témoins obtenus selon la technologie traditionnelle. Cette différence initiale qui se maintient jusqu'au $21^{\mathrm{e}}$ jour d'affinage a pour origine l'élévation du pouvoir tampon des rétentats, due à l'enrichissement en sels phosphocalciques (BRULÉ et al., 1974) concentrés en même temps que la caséine à laquelle ils sont liés. Cette élévation du pouvoir tampon des préfromages Saint-Paulin est telle que le $\mathrm{pH}$ des fromages qui en sont issus reste supérieur à celui mesuré dans les fromages conventionnels correspondants, bien que la concentration en bactéries lactiques soit 10 fois supérieure. La lyse bactérienne naturelle qui se produit dans les tous premiers jours de la maturation des pâtes traditionnelles - et nos observations sur ce type de fromage sont conformes à celles faites antérieurement par Ducastelle et Lenoir (1965) - n'est pas observée dans les fromages de lait ultrafiltré. Le maintien en «survie » des cellules bactériennes dans les pâtes fromagères issues de la technologie MMV pourrait donc avoir pour conséquence la non-libération pendant les premiers temps de l'affinage des endo-enzymes par- 


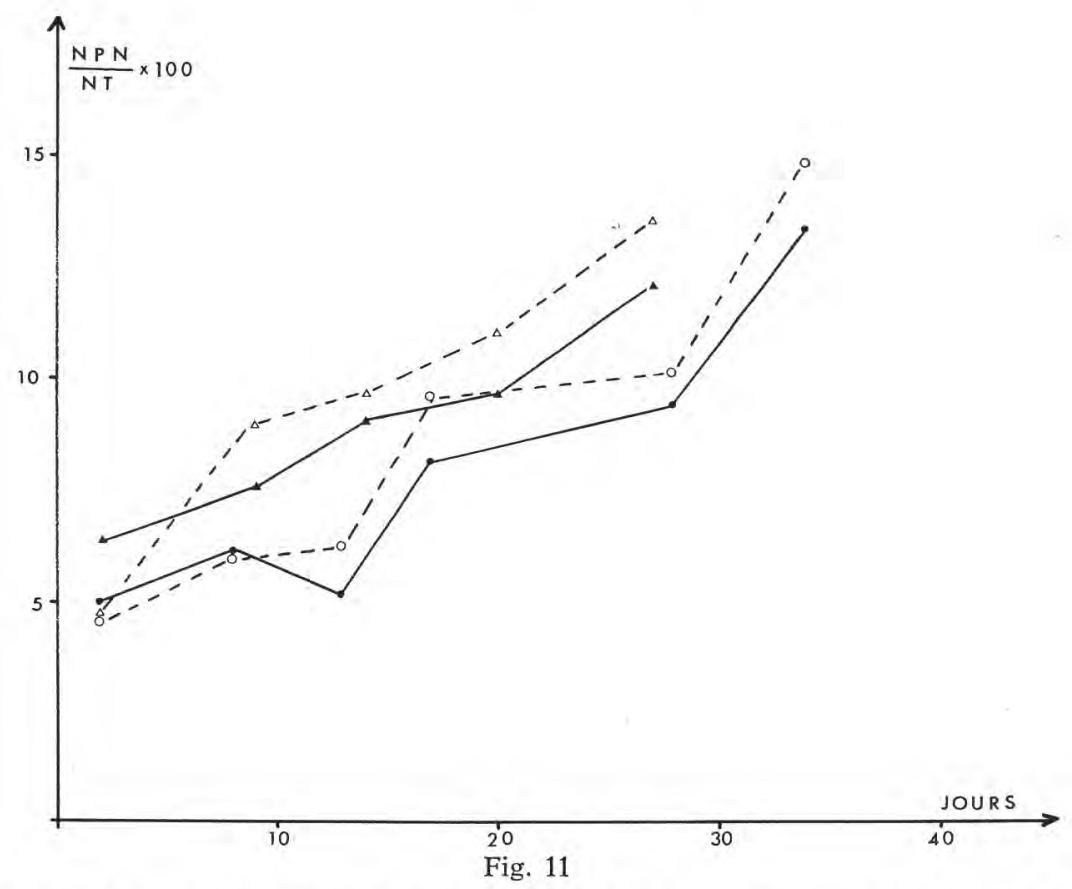

Evolution de la fraction azotée non protéique (NPN) de fromages de type St-Paulin au cours de l'affinage :

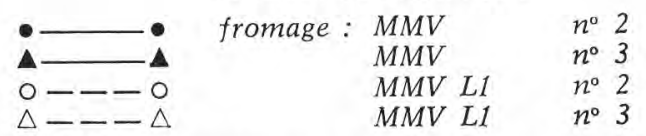

Evolution of soluble nitrogen content in St-Paulin cheeses added or not with lysozyme during ripening.

ticipant au développement de la saveur et de l'arôme par action sur les composants lipidiques et protéiques du milieu. Cette hypothèse n'est pas contredite par l'évolution du rapport NPN/NT (fig. 5). En effet, compte tenu que les teneurs en NPN sont plus importantes dans les. fromages Saint-Paulin MMV en début d'affinage du fait de leur composition originelle - ils contiennent la quasi-totalité du caséinomacropeptide libéré par action de la présure sur la caséine Kappa puisque l'égouttage ne représente que 7-9 p. 100 du poids du préfromage (GOUDEDRANCHE et al., 1980) - la vitesse de protéolyse de ces pâtes est significativement plus faible pendant toute la durée d'affinage étudiée.

Le protocole de fabricaton suivi pour la fabrication des fromages Camembert en technologie MMV conduit à des fromages dont l'évolution du $\mathrm{pH}$ et celle de la flore mésophile sont comparables à celles des fromages issus de la technologie conventionnelle. Un tel résultat est conforme à ce qui pouvait être attendu puisque le protocole suivi a été développé dans le but d'atteindre dans ce type de pâte une minéralisation calcique voisine de celle observée dans les fromages témoins (GoudedRANCHE et al., 1979). 
L'addition de lysozyme aux préfromages Saint-Paulin conduit à une diminution de la flore aérobie mésophile, aussi bien les premiers jours de l'affinage que dans les semaines qui suivent. Les valeurs de population dénombrées se rapprochent de celles observées dans les fromages conventionnels. Cette évolution des dénombrements dans les préfromages Saint-Paulin additionnés de lysozyme est à rapprocher de l'augmentation constatée de la protéolyse au cours de l'affinage (fig. 11) et de l'amélioration des qualités organoleptiques des fromages qui en sont issus, ces derniers restant toutefois légèrement inférieurs en goût et en flaveur aux produits traditionnels. De par son action lytique sur les bactéries lactiques présentes dans les caillés de fromagerie, le lysozyme pourrait donc avoir un effet bénéfique dans la conduite, voire l'accélération de l'affinage aussi bien des fromages à pâte demi-dure que des fromages à pâte molle de type Camembert. Les observations réalisées dans cette étude, jointes aux résultats obtenus récemment par THAPON et BRULÉ (1986), conduisent à penser que la cinétique d'action du lysozyme pourrait être la suivante : lors de l'addition au préfromage, la totalité du lysozyme serait complexée par les caséines et la matière grasse présentes; dès le saumurage, il y aurait relargage du lysozyme dans la phase aqueuse des fromages sous l'effet de l'accroissement de la force ionique et la teneur en enzyme actif dans la phase aqueuse atteindrait une valeur suffisante pour provoquer, soit la lyse cellulaire partielle, soit la formation de protoplastes se lysant progressivement, mécanismes aboutissant l'un et l'autre à la libération des endoenzymes. La présence de protéines du lactosérum à l'état natif dans les caillés MMV pourrait également accroître l'activité lytique du lysozyme (MıHAIL, 1983) une fois l'enzyme décomplexée des micelles de caséine.

Les déterminations réalisées lors de l'addition de lysozyme aux préfromages Camembert ne mettent pas en évidence de variations significatives de la population bactérienne dans les fromages au cours de leur affinage. L'effet significativement favorable de l'addition de lysozyme sur les qualités organoleptiques doit donc avoir pour origine des mécanismes complexes où interviennent certes les enzymes exo et intracellulaires des bactéries lactiques (pour une part nettement plus faible que dans le cas des pâtes Saint-Paulin), mais, surtout, les enzymes excrétées par la moisissure de surface dont le rôle est dominant pour l'obtention des textures et des flaveurs recherchées. Il est probable aussi que la remontée rapide du $\mathrm{pH}$ dans ce type de pâte modifie l'activité du lysozyme vis-à-vis des parois cellulaires.

S'il n'y a pas ajustement préalable de la minéralisation calcique, la protéolyse observée dans les fromages Saint-Paulin issus de la technologie MMV est plus faible que celle existant dans les fromages conventionnels. Il en résulte des qualités organoleptiques amoindries, ce qui est dommageable pour le consommateur. L'addition de lysozyme aux préfromages Saint-Paulin et Camembert semble être un moyen intéressant pour accélérer et diriger l'affinage des fromages issus de cette technologie afin de rapprocher leurs qualités organoleptiques de celles des produits traditionnels. La dose à ajouter serait de l'ordre de 0,5 à $1,0 \mathrm{~g} / \mathrm{kg}$ de préfromage, soit un coût non négligeable au $\mathrm{kg}$ de fromage. Il est possible qu'une dose quantitativement plus faible produise les mêmes effets sur les qualités organoleptiques. Outre cette recherche de la dose minimale efficace, bien des travaux restent encore à mener pour déterminer les conditions optimales d'utilisation du lysozyme en fromagerie MMV et en comprendre les mécanismes d'action. II serait également intéressant d'étudier les effets de l'addition du lysozyme sur l'affinage des fromages issus de technologies traditionnelles. Enfin, les expéri- 
mentations réalisées dans le cadre de cette étude sur l'addition de lysozyme à des levains mésophiles et thermophiles, qui ont abouti à des résultats négatifs, mériteraient d'être reprises car la possible utilisation de protoplastes comme «sacs d'enzymes» destinés à accélérer ou à régulariser l'affinage nous semble une voie technologique prometteuse nécessitant une recherche approfondie.

Reçu le 25-11-1985.

Accepté pour publication le 4-2-1986.

\section{Références bibliographiques}

Brulé G., Maubois J.L., Fauquant J., 1974. Etude de la teneur en éléments minéraux des produits obtenus lors de l'ultrafiltration du lait sur membrane. Lait, 54, (539-540), 600-615.

Ducastelle A., Lenoir J., 1965. Contribution à l'étude de la flore microbienne du fromage de type Saint-Paulin. Lait, 45, (447), 371-378.

Ducruet P., Maubois J.L., Goudedranche H., Pannetier R., 1981. Eléments de fabrication de fromages à pâte demi-dure et à ouverture propionique selon le procédé MMV. Tech. lait., (957), 13-16.

Goudedranche H., Ducruet P., Maubors J.L., 1979. Résultats non publiés.

Goudedranche H., Maubois J.L., Ducruet P., Mahaut M., 1980. Utilization of the new mineral UF membranes for making semi-hard cheeses. Desalination, 35, 243-258.

Guilloteau J.C., 1981. Procédé de fabrication de fromages frais et de fromages à pâte molle à croûte moisie. Brevet français $\mathrm{n}^{\circ} 8105157$.

Maubois J.L., Mocouot G., Vassal L., 1969. Procédé de traitement du lait et de sous-produits laitiers. Brevet français $\mathrm{n}^{\circ} 2052121$.

MinaIL C., 1983. Interactionea tizozimului uman cu unele componente din lapte. St. Cerc. Biochim., 26, (1), 50-57.

Thapon J.L., Brulé: G., 1986. Effets du pH et de la force ionique sur l'affinité lysozymecaséines. Lait, 66, (1), 19-30.

VASSAL L., 1984. Analyse sensorielle des Emmentals fabriqués à partir de lait ultrafiltré à à la ferme. Rapport Commission C.N.E.R.N.A. 